\title{
REVIEWS
}

\section{Osteoporosis: whose problem is it?}

\author{
Anthony D Woolf
}

Osteoporosis is one of the major diseases that affects the aging population with enormous cost in morbidity and mortality to the subject and to the community in demands on the provision of care. The growing financial costs are becoming increasingly apparent in a cost conscious health care service. There were 43230 hip fractures in England in 1985, ${ }^{1}$ and average hospital inpatient stay was 30 days. Excess mortality in the subsequent 12 months is up to $20 \%,{ }^{2}$ and survivors often have permanent disability and dependency-50\% no longer walking independently. ${ }^{3}$ The hospital costs of these fractures were estimated at $£ 160$ million in $1987-8,{ }^{4}$ but this omits the costs of community care. It also ignores the indirect cost in suffering to these patients on long waiting lists for joint replacement surgery that has been delayed because orthopaedic resources cannot deal adequately with both them and the unpredictable workload of trauma. The morbidity of vertebral fractures is not always so obvious. Loss of height and stoop has long been dismissed as normal aging. It is true that it is common in the elderly$22.5 \%$ of 70 year old women having a vertebral fracture, ${ }^{5}$-but it should not be considered inevitable or of no consequence as it does produce symptoms, is preventable, ${ }^{6}$ and is treatable. $^{7}$

Despite this high prevalence of clinical osteoporosis-that is, those who have sustained a fracture, recent surveys have shown that many clinicians seldom diagnose it or recognise those at risk. Public awareness has been raised more recently by the National Osteoporosis Society with the support of patients, government, and industry, who are aware of the scale of the problem. For any treatment and preventive campaign to be effective it does not only need public awareness but also interested and informed clinicians and researchers. Why is there this lack of awareness? It is not a new disease. It has been demonstrated in neolithic skeletons ${ }^{8}$ and was probably first described in the sixth century AD. In 1824 Astley-Cooper noted that bones become "thin in their shell and spongy in their texture' in old age and that fractures of the neck of femur often follow moderate trauma, ${ }^{1}$ and in 1940 Albright suggested that the menopause might be an important risk factor for osteoporosis. ${ }^{9}$ Until recently, however, those interested in osteoporosis have been a small group from varied disciplines as there are few specialist bone units. Is this the cause of lack of awareness and knowledge and how can it be improved? Expertise is needed in all teaching centres and district hospitals not only to manage patients but also to educate medical students and family practitioners.

\section{Whose problem is it?}

Patients will consult different specialties about osteoporosis-the family practitioner, the gynaecologist about hormone replacement therapy, the rheumatologist about back pain, the orthopaedic surgeon after a fracture, the geriatrician about loss of independence, but it surely would be of benefit if there were specialists who saw patients from mid-life for prevention, through to old age and the treatment of their established osteoporosis. These will be from various disciplines, but would it be better if one specialist group took a major interest? The gynaecologist is unlikely to be interested in chronic back pain and adaptations to the home to help the elderly person remain independent. Orthopaedic surgeons, despite the proportion of their workload that is a direct consequence of osteoporosis, are unlikely to wish to monitor long term treatments or develop preventive strategies. The rheumatologist will predominantly see patients with symptoms or disabilities resulting from their osteoporosis. At this stage bone mass cannot be restored and it is uncertain whether bone strength can be significantly improved once lost. Restoration of the microarchitecture must be shown for this to be convincingly proved. The patient does, however, have chronic pain and disability, which rheumatologists are experienced at treating. Several analogies can be made between the development of rheumatology and current questions about osteoporosis, providing further reasons why rheumatologists may be interested in the assessment of risk and prevention of osteoporosis. An understanding of osteoarthritis has been impaired because it too has been dismissed as an inevitable part of aging. Further analogies are: (a) the prevention of erosive changes of rheumatoid arthritis with disease modifying antirheumatic drugs rather than just treating late disability, and the role of hormone replacement therapy in prevention of osteoporosis; $(b)$ identification of those patients with rheumatoid arthritis who will have a poor outcome and need early aggressive treatment ${ }^{10}$ and targetting of those most at risk of osteoporosis; ${ }^{11} 12(c)$ the lack of data supporting the long term benefits of disease modifying antirheumatic drugs ${ }^{13}$ despite their extensive use by a generation, and the favourable short term results for treatment of osteoporosis with calcitonin and diphosphonates ${ }^{7}$ but lack of long term data necessary fully to establish efficacy. 
The rheumatologist should therefore be well motivated to prevent rather than just treat, to try to target prevention at those most at risk, and to report the long term effects of treatment to learn if fractures can be prevented.

\section{The rheumatologist's role}

Rheumatologists in the United Kingdom have largely kept their interest to the other side of the synovial membrane, though in Europe, and more recently in the USA, osteoporosis and bone disease is an established part of rheumatology. This is now changing in the United Kingdom. At a recent meeting of the British Society for Rheumatology it was established that there is sufficient interest. Many rheumatologists are seeing patients concerning treatment and are also participating in prevention of osteoporosis and have access to methods of assessing bone mass. More importantly, are rheumatologists adequately trained and resourced for this potential workload if they choose to become involved? How can other advantages be gained from a stronger participation? A British Society for Rheumatology Osteoporosis Interest Group has been established and a workshop was recently held to look at the questions which need to be answered about assessment, prevention, and treatment of osteoporosis and how this can be attempted by an interested group of rheumatologists. The points made are discussed here. It was recognised that there is an inherent weakness in the collection of prospective data unless clear questions are being asked and appropriate control data are simultaneously collected.

\section{Questions to be answered}

Among the many epidemiological questions which remain to be answered about osteoporosis, the most important is the choice of preventive strategy. Although hormone replacement therapy represents a potent means of reducing bone loss and preventing fractures, there are concerns about the application of this measure to all postmenopausal women. Much attention has thus been focused on methods whereby those women at highest risk of developing osteoporosis might be identified. Three broad approaches have been used: bone density measurements, risk factor profiles, and biochemical assessment of bone turnover. Bone density correlates with bone strength, and longitudinal data are now available to support an association between bone density and fracture risk. ${ }^{14}$ This association does not seem to be sufficiently strong, using currently available methodology, to justify widespread bone density screening. ${ }^{15}$ Historical risk factor profiles have also generally lacked predictive value for this purpose, ${ }^{11} 12$ and biochemical assessment remains the subject of much research. Collection of data on large cohorts of well characterised subjects could provide information as to whether some of these methods might be used in combination to better effect.

There are various methods of bone mass assessment and the most popular and reproducible method at present is dual energy $x$ ray absorptiometry. ${ }^{16}$ There are, however, differences between results from commercial machines which need to be resolved to allow comparison of accumulated data. Anterior/ posterior views of the lumbar spine are usually assessed, but this includes calcification of the aorta and osteophytosis of apophysial joints. This can be excluded with lateral scans, though only one or two lumbar vertebrae can be assessed reliably by this method because of overlap by ribs and pelvis. The proximal femur is assessed at different sites and it remains to be established which site correlates best with fracture risk. Although this method appears reproducible and accurate in normal subjects, the limited agility of patients with osteoporosis or arthritis must be assessed for its effect on these. The principal question to be answered about bone mass assessment is its sensitivity and specificity for assessing future risk of fracture. Can a normogram be constructed? The efficacy of treatments on rate of bone loss can now be accurately assessed, but this is an adjunct to, not a substitute for, fracture data.

An alternative approach to assessing rate of bone loss is by biochemical markers of bone resorption and formation. There are several biochemical markers available for the study of bone turnover in health and disease. These can be broadly classified into two categories: those primarily indicating bone synthesis and those primarily studying bone resorption. The first category includes estimation of serum levels of osteoclast products, such as osteocalcin and alkaline phosphatase, whereas the second includes estimation of circulating levels of the osteoclast marker tartrate resistant acid phosphatase or urinary concentrations of catabolic products of collagen, such as hydroxylysine glycosides, hydroxyproline, and, more recently, the pyridinium cross links.

Serum osteocalcin is probably the best biochemical marker currently available for the study of bone formation. ${ }^{17}$ Hydroxyproline has been widely used in the study of metabolic bone disease,$^{18}$ but it is not solely a marker of skeletal collagen degradation as it may also arise from the collagens of skin and soft tissue; it may also arise from the breakdown of procollagen peptides released during collagen synthesis. The sensitivity of this marker is further reduced by the ability of the liver to metabolise up to $90 \%$ of the plasma pool. Recently, much work has centred on the use of pyridinium cross links of collagen in the study of metabolic and inflammatory bone disease, and there is now sufficient evidence to indicate that these components, especially the cross links specific to bone collagen (deoxypyridinoline), are more specific and more sensitive markers of bone degradation than those used previously. ${ }^{19} 20$ Long term studies are needed to assess whether these measurements on a single occasion will predict change in bone mass over a period of time.

Histomorphometry of iliac crest bone biopsy specimens is used to assess the structural changes that accompany age related bone loss. Trabecular bone loss may result from trabecular 
thinning with overall trabecular architecture preserved or there may be erosion through and removal of trabeculae leading to a much greater loss of connectedness of the bone structural pattern. ${ }^{21}$ Loss of the connectedness is associated with greater reduction in bone strength than trabecular thinning, ${ }^{22}$ and although thinning may be reversible, ${ }^{23}$ it is unknown whether trabecular connectedness can be restored. There is a cycle of bone cell activity with osteoclasts initially resorbing bone followed by osteoblasts depositing osteoid, which is subsequently calcified and mineralised. Any imbalance of this cycle will result in net bone loss. $^{24}$ These processes can be assessed by histomorphometry with double tetracycline labelling giving dynamic indices of formation and can be used to study differences between patients with and without fracture, between different types of osteoporosis, and to monitor the response to treatment. Disadvantages are that it is invasive, time consuming, and normal variation can be great, making the method unsuitable for routine assessment.

Prevention of osteoporosis with hormone replacement therapy has been established as effective, ${ }^{6}$ and many studies are currently further examining safety and the risk/benefit ratio. Its role in older women with osteoporosis is not established. Hormone replacement therapy is, however, not acceptable to many women and alternative preventive and therapeutic approaches are needed. The role of calcium is much debated, ${ }^{25}$ and controlled prospective data are still needed to establish its effectiveness. If it was only $1 \%$ more effective than placebo in reducing the incidence of fractures it would prevent more than 1000 fractures a year in Britain. ${ }^{26}$ Formal exercise programmes reduce bone loss rates, ${ }^{27-29}$ but the effect of a more realistic general increase in physical activity on bone mass and fracture rate remains to be demonstrated. Other treatments, such as calcitonin, diphosphonates, anabolic steroids, and fluoride, ${ }^{7}$ have been shown to reduce bone loss in normal postmenopausal women or in those with established osteoporosis, but long term benefits on bone mass and fracture rates remain to be shown. The initial favourable open studies of fluoride $^{23} 30$ have now been clouded by the contradictory results of the NIH sponsored, controlled, prospective study ${ }^{31}$ and the collective experience of fluoride treatment in several centres in France, ${ }^{32}$ which may relate to differences in dose used. Demonstration of fracture prevention at appendicular and axial sites is essential to prove a clinically relevant response as restoration of bone mass does not necessarily reflect restoration in strength unless trabecular connectedness is restored. ${ }^{21}{ }^{22}$ Reduction in further fractures has been shown in recent studies of etidronate, ${ }^{33}{ }^{34}$ but the numbers of subjects are small when considering the unpredictable event of fracture. ${ }^{35}$ It is often difficult to compare studies of the same therapeutic agent as a variety of regimens have been used. Diphosphonates have been given either continuously, as part of coherence therapy ${ }^{24}$ or, most recently, intermittently for two weeks every three months. ${ }^{33} 34$ Different types of calcitonin at different doses have been shown to reduce bone resorption. The manufacturer's recommended dose of salmon calcitonin is $\mathbf{1 0 0}$ IU daily, but it has not been established whether this offers any long term benefit over more economical lower doses. Some treatments may be better targeted at either low formation or high resorption osteoporosis as determined by biochemical markers. These additional variables and the drop out rates of any long term study increase the numbers who would need to be assessed. These points emphasise the need for large long term controlled prospective studies using standardised treatment regimens to obtain a full assessment and comparison of these emerging treatments for osteoporosis.

A more relevant question to rheumatologists is steroid induced osteoporosis and its prevention. Although it was first described by Cushing in his original description of adrenal hyperactivity, there are many unanswered questions. ${ }^{36}$ Is there a safe dose? When is bone loss greatest? Do all steroids cause bone loss? Is it preventable or reversible? In this predictable form of osteoporosis there should be clearer answers-these could be supplied if the appropriate large long term studies were established.

Patients would clearly benefit if there was greater clinical awareness and understanding of osteoporosis. It is argued that this might be best achieved if more doctors had specialist knowledge and if osteoporosis formed part of the routine training and clinical practice of a specialty such as rheumatology. It would then be their role to teach future medical students. Many of the outstanding questions can be approached by the concerted effort proposed and a protocol is being developed initially for steroid induced osteoporosis. If this approach receives sufficient interest and support then further protocols could be developed in an attempt to answer the other outstanding questions.

The workshop of the Osteoporosis Interest Group was held at the Royal Cornwall Hospital, Truro in March 1990. Participants were D Barlow, A Bhalla, D Black, C Cooper, S Doherty, A Freemont, R Gray, D Pye, A D Woolf.

1 Griffin J. Osteoporosis and the risk of fracture. London: Office of Health Economics, 1990.

2 Cummings S R, Kelsey J L, Nevitt M C, O'Dowd K J. Epidemiology of osteoporosis and osteoporotic fractures. Epidemiol Rev 1985; 7: 178-208.

3 Miller $\mathrm{C} \mathrm{W}$. Survival and ambulation following hip fracture. F Bone foint Surg [Am] 1978; 60: 930-4.

4 Fractured neck of femur: prevention and management. London: Royal College of Physicians, 1989.

5 Jensen G F, Christiansen C, Boesen J, Hegedüs V, Transbøl I. Epidemiology of postmenopausal spinal and long bone fractures: a unifying approach to postmenopausal osteofractures: a unifying approach to pos
porosis. Clin Orthop 1982; 166: 75-81.

6 Roche $M$, Vessey $M$. Hormone replacement therapy in the menopause: risks, benefits and costs. In: Smith $\mathbf{R}$, ed. Osteoporosis 1990. London: Royal College of Physicians, 1990: 189-98.

7 Reeve J. Restoring trabecular bone mass in established osteoporosis. In: Smith R, ed. Osteoporosis 1990. London: Royal College of Physicians, 1990: 141-55.

8 Woolf A D, Dixon A St J. Osteoporosis: a clinical guide. London: Martin Dunitz, 1990.

9 Albright F, Smith P H, Richardson A M. Postmenopausal osteoporosis: its clinical features. $\mathcal{F} A M A$ 1941;116:2464-74.

10 Woolf A D, Hall N D, Kantharia B, Maymo J, Goulding N J, Maddison P J. Predictors of 5 years outcome of early synovitis. $B r \mathcal{F}$ Rheumatol 1986; 25 (suppl 2): 24.

11 Stevenson J C, Lees B, Devenport M, Cost M P, Ganger K F. determinants of bone density in normal women: risk factors for future osteoporosis. $B M \mathcal{F} 1989 ; 298$ : 924-8. 
12 Cooper C, Shah S, Hand D J, et al. Screening for osteoporosis using individual risk factors. Osteoporosis Int. In press.

13 Iannuzzi L, Dawson N, Zein N, Kushner I. Does drug therapy slow radiographic deterioration in rheumatoid arthritis? N Engl f Med 1983; 309: 1023-8.

14 Cummings S R, Black D M, Nevitt M C, et al. The study of osteoporotic fractures research group: appendicular bone density and age predict hip fracture in women. $J A M A$ density and age pred

15 Raffle A E, Cooper C. Bone density screening for osteoporosis. Lancet 1990; 336: 242.

16 Mazess R B, Sorenson J A, Hanson J A, Collick B D, Smith $S W$. Dual-energy $x$-ray absorptiometry (DEXA). In: Ring E F J, Evans W D, Dixon A S, eds. Osteoporosis and bone mineral measurement. York: Institute of Physical Sciences in Medicine, 1989; 62-71.

17 Brown J P, Delmas P D, Malaval L, Edouard C, Chapuy M C, Meunier P J. Serum bone Gla protein: a specific marker for bone formation in postmenopausal osteoporosis. Lancel 1984; i: 1091-3.

18 Kivirikko K I. Urinary excretion of hydroxyproline in health and disease. Int Rev Connect Tissue Res 1970; 5: 93-163.

19 Black D, Farquharson C, Robins S P. Excretia of pyridinium crosslinks of collagen in ovarectomised rats as urinary markers of increased bone resorption. Calcif Tissue In 1989; 44: 343-7.

20 Robins S P, Black D, Paterson C, Reid D M, Duncan A, Seibel $M$ J. Evaluation of urinary hydroxypyridinium crosslink measurements as resorption markers in metabolic bone diseases. Eur $\mathcal{F}$ Clin Invest 1991; 21: 310-5.

21 Compston J E. Structural mechanisms of trabecular bone loss. In: Smith R, ed. Osteoporosis 1990. London: Royal College of Physicians, London, 1990: 35-43.

22 Pugh J W, Rose R M, Radin E L. Elastic and viscolastic properties of bone. $\mathcal{F}$ Biomech 1973; 6: 475-85.

23 Briancon D, Meunier P J. Treatment of osteoporosis with fluoride, calcium and vitamin D. Orthop Clin North Am 1981; 12: 629-48.

24 Parfitt A M. The coupling of bone formation to bone resorption: a critical analysis of the concept and of its relevance to the pathogenesis of osteoporosis. Metab Bone Dis Relat Res 1984; 4: 1-6.

25 Kanis J A, Passmore R. Calcium supplementation of the diet I. BMF 1989; 298: $137-40$.

26 Kanis J A, Passmore R. Calcium supplementation of the diet II. $B M \mathcal{F} 1989 ; 298: 205-8$.

27 Chow R, Harrison J E, Notarius C. Effect of two randomised exercise programmes on bone mass of healthy postmenopausal women. $B M \mathcal{F}$ 1987; 295: 1441-4.

28 Dalsky G P, Stocke K S, Ehsani A A, Slatopolski E, Lee W C, Birge S T Jr. Weight-bearing exercise training and lumbar bone mineral content in postmenopausal women. Ann Intern Med 1988; 108: 824-8.

29 Smith E L, Gilligan C, McAdam M, Ensign C P, Smith P E Deterring bone loss by exercise intervention in premenopausal and postmenopausal women. Calcif Tissue Int 1989; 44: $312-21$.

30 Riggs B L, Seaman E, Hodgson S F, Taves D R, O'Fallon W $M$. Effect of the fluoride/calcium regime on vertebral fracture occurrence in postmenopausal osteoporosis: comparison with conventional therapy. N Englf Med 1982; 306: 446-50.

31 Riggs B L, Hodgson S F, O'Fallon W M, et al. Effect of fluoride treatment on the fracture rate in postmenopausal fluoride treatment on the fracture rate in postmenopausal
women with osteoporosis. $N$ Engl F Med 1990; 322: 802-9.

32 Mamelle N, Meunier P J, Dusan R, et al. Risk-benefit ratio of sodium fluoride treatment in primary vertebral osteoporosis. Lancet 1988; ii: 361-5.

33 Storm T, Thamsborg G, Steiniche T, Genant H K, Sorensen $\mathrm{O} \mathbf{H}$. Effect of intermittent cyclical etidronate therapy on bone mass and fracture rate in women with postmenopausal osteoporosis. N Engl f Med 1990; 332: 1265-71.

34 Watts N B, Harris S T, Genant $\mathbf{H ~ K}$, et al. Intermittent cyclical etidronate treatment of postmenopausal osteoporosis. N Engl f Med 1990; 323: 73-9.

35 Kanis J A. Treatment of osteoporotic fracture. Lancet 1984; i: 27-33.

36 Woolf A D. Steroid induced osteoporosis: questions to be answered. In: Ring E F J, Evans W D, Dixon A S, eds. Osteoporosis and bone mineral measurement. York: Institute of Physical Sciences in Medicine, 1989: 271-7. 